\title{
Apport de la technologie " Cone beam" dans le repérage de l'artère maxillaire au niveau de la paroi latérale du sinus : intérêt en chirurgie implantaire.
}

\author{
Bally $\mathbf{J}^{1}$, Curien $\mathbf{R}^{2}$, Hodez $C^{3}$, Sourdot $A^{1}$, Le $A^{1}$, Bravetti $P^{1}$ \\ ${ }^{1}$ Faculté d'Odontologie, Département de Chirurgie et Pathologie buccales, Nancy, France \\ ${ }^{2}$ Service d'Odontologie, CHU, Nancy, France \\ ${ }^{3}$ Médecin radiologue, cabinet de Radiologie et d'Imagerie médicale, Nancy, France \\ ballyjulien@hotmail.com
}

Une hauteur osseuse insuffisante, notamment dans le secteur du maxillaire postérieur, constitue un obstacle à la bonne mise en place d'implant dentaire. Désormais, les techniques d'élévation du plancher sinusien permettent d'augmenter la hauteur de crête osseuse résiduelle. L'abord latéral $\mathrm{du}$ sinus avec réalisation d'une fenêtre osseuse latérale représente l'une des techniques chirurgicales à disposition. Cependant sa réalisation nécessite une parfaite connaissance de l'anatomie du sinus et de sa vascularisation. L'étude de Solar et al. (1999) décrit notamment un vaisseau endo-osseux, provenant de l'artère alvéolaire postérieure, et cheminant dans la paroi latérale du sinus. Aussi, la situation des branches de cette artère peut influencer le choix de la localisation de la fenêtre latérale.

Le but de cette étude est d'observer la répartition des branches endo-osseuses de l'artère alvéolaire dans le secteur de la fenêtre latérale. Dans ce cadre, la technologie dite "à faisceau conique", équivalent français de cone beam, ou encore "Cone Beam Computed Tomography" (CBCT), devrait permettre une visualisation beaucoup plus systématique de cette artère par rapport aux examens tomodensitométriques classiques. En effet, les voxels isotropes permettent, contrairement au scanner, d'obtenir des images dont la résolution spatiale est la même quelle que soit l'orientation des coupes. Un "3D Accuitomo" a été utilisé pour cette étude. Toutes les machines ne donnant pas des résultats équivalents en matière de résolution spatiale, il est important de souligner que le volume étudié est composé de voxels isotropes de $125 \mu$ d'arête. La petite taille des voxels confère à cette machine des images dont la résolution spatiale est la meilleure actuellement disponible au sein des technologies "à faisceau conique". Les mesures sont réalisées directement sur la console informatique grâce à l'outil de mesure disponible sur la visionneuse 3D fournie avec chaque examen. Sur 110 examens cone beam réalisés pour un bilan pré-implantaire dans le secteur maxillaire droit, en position 15,16;65 sont choisis pour l'étude. Dans les cas où l'artère a pu être observée, des mesures sont réalisées pour déterminer les distances entre le centre de l'artère et la crête alvéolaire, et le plancher sinusien.

Dans notre étude, l'artère est visible dans $75,38 \%$ des cas. La distance moyenne entre l'artère et le sommet de la crête est de $13,82 \mathrm{~mm}( \pm 2,51 \mathrm{~mm})$ et de $9,07 \mathrm{~mm}( \pm 2,1 \mathrm{~mm})$ avec le plancher sinusien.

Avec cette étude, on peut se rendre compte de l'intérêt de la technologie cone beam en matière de visualisation de ce passage vasculaire. En effet, dans l'étude d'Elian et al. (2007), l'artère est retrouvée sur $51,4 \%$ des clichés étudiés. D'autre part, la mesure de la distance entre l'artère et le plancher sinusien permet au praticien de la visualiser de manière très précise. Cette artère peut être à l'origine de complications hémorragiques per-opératoires. Même si ces saignements sont généralement maîtrisés facilement, ils peuvent gêner la visualisation de la membrane de Schneider, ainsi que la mise en place de matériaux de comblement. On a ici une parfaite illustration de ce que la technologie cone beam, peut apporter dans le domaine de la chirurgie préimplantaire. 